5. Graves S, Stenos J. Rickettsioses in Australia. Ann N Y Acad Sci. 2009;1166:151-5. http://dx.doi.org/10.1111/j.17496632.2009.04530.x

6. Unsworth NB, Stenos J, Graves SR, Faa AG, Cox GE, Dyer JR, et al. Flinders Island spotted fever rickettsioses caused by "marmionii" strain of Rickettsia honei, eastern Australia. Emerg Infect Dis. 2007;13:566-73. http://dx.doi.org/10.3201/eid1304.050087

Address for correspondence: Rikki M.A. Graham, Public Health Microbiology, Public and Environmental Health, Department of Health, Forensic and Scientific Services, PO Box 594, Archerfield, QLD 4108, Australia; email: rikki.graham@health.qld.gov.au

\title{
Chlamydia trachomatis Biovar L2 Infection in Women in South Africa
}

\section{Remco P.H. Peters, ${ }^{1}$ Ronan Doyle, ${ }^{1}$ Mathys J. Redelinghuys, James A. McIntyre, Georges M. Verjans, Judith Breuer, Marleen M. Kock}

Author affiliations: University of Pretoria, Pretoria, South Africa (R.P.H. Peters, M.J. Redelinghuys, M.M. Kock): Maastricht University Medical Centre, Maastricht, the Netherlands (R.P.H. Peters); Anova Health Institute, Johannesburg, South Africa (R.P.H. Peters, J.A. McIntyre); University College London, London, United Kingdom (R. Doyle, J. Breuer); University of Cape Town, Cape Town, South Africa (J.A. Mclntyre); Erasmus Medical Centre, Rotterdam, the Netherlands (G.M. Verjans); National Health Laboratory Service, Pretoria (M.M. Kock)

DOI: https://doi.org/10.3201/eid2311.170758

We detected Chlamydia trachomatis biovar L2 in vaginal swab specimens of 7 women with vaginal discharge in South Africa. Whole-genome sequencing directly from clinical specimens identified a closely related cluster of strains. The clinical role of this infection in the context of syndromic management should be clarified.

I nfection with Chlamydia trachomatis biovar $\mathrm{L}$ is known as lymphogranuloma venereum (LGV). This infection usually presents as genital ulcers, followed by an invasion of the lymphatic system resulting in buboes, painful swelling of lymph nodes (1). In the past 2 decades, another

${ }^{1}$ These authors contributed equally to this article. manifestation of LGV has emerged in North America and Europe: rectal LGV infection causing proctocolitis among men who have sex with men (MSM) (1). In this population, urethral LGV also occurs (2).

There have been only sporadic reports of rectal and genital LGV infection in women living in the industrialized world $(3,4)$. Cross-sectional studies from France, Switzerland, and the Netherlands did not detect biovar L in specimens from women with genital or rectal C. trachomatis infection $(1,5-7)$. Because lymphatic manifestation has become relatively rare, LGV infection is considered an outbreak mainly among MSM in Europe and North America (1). Lymphatic LGV is endemic to Africa, but before our study, it was unknown whether C. trachomatis biovar $\mathrm{L}$ infections occurred in women in Africa. Thus, we determined the prevalence of this infection in South Africa.

To determine whether genital C. trachomatis biovar $\mathrm{L}$ infections occur in women living in South Africa, we analyzed 82 DNA samples extracted from vaginal swab specimens that were positive by a molecular detection assay for $C$. trachomatis infection at the Department of Medical Microbiology at the University of Pretoria. The Faculty of Health Sciences Research Ethics Committee at the University of Pretoria approved the studies in which these specimens were collected. These swab specimens had been collected during 2012-2016 from women attending different healthcare settings: a mobile health clinic in rural Mopani District $(n=52)$ and 3 departments at the academic hospital in Pretoria: obstetrics and gynecology clinic $(\mathrm{n}=14)$, antiretroviral treatment clinic $(\mathrm{n}=10)$, and sexually transmitted infection (STI) clinic $(n=6)$. We assessed the presence of LGV in these genital specimens by using specific PCRs for C. trachomatis serovar L and serovar L2b (8). For positive PCR results, we confirmed the diagnosis by conducting whole-genome sequencing (WGS) of $C$. trachomatis directly from the clinical specimen as described elsewhere (9).

Whereas C. trachomatis biovar $\mathrm{L}$-specific PCR showed positive results for 7 specimens obtained from women at the antiretroviral treatment $(n=5)$ and STI $(n=$ 2) clinics in Pretoria, we did not detect LGV in any of the 52 specimens from women in Mopani District. All PCR test results for serovar L2b were negative. The 7 women with genital LGV all had vaginal discharge and were co-infected with another STI (Table).

WGS confirmed LGV (ompA sequence identical to those of the $C$. trachomatis L2 434/BU reference strain) in 4 cases with good mean read depth $(\geq 12)$ and high genome coverage (>98\%). The 4 sequences clustered well with the L2 sequences previously published and away from L1 and L2b sequences. For 1 specimen, the mean read depth 
Table 1. Characteristics of 7 women with vaginal discharge and a positive PCR result for Chlamydia trachomatis biovar L, Pretoria, South Africa, 2012-2016*

\begin{tabular}{|c|c|c|c|c|c|c|}
\hline $\begin{array}{l}\text { Patient } \\
\text { ID }\end{array}$ & $\begin{array}{c}\text { Healthcare } \\
\text { setting }\end{array}$ & HIV status & Co-infection & C. trachomatis WGS result & $\begin{array}{c}\text { Mean read } \\
\text { depth }\end{array}$ & $\begin{array}{c}\text { Genome } \\
\text { coverage, \% }\end{array}$ \\
\hline 1 & ART clinic & Positive & Trichomonas vaginalis & L2 confirmed & 41 & 99.5 \\
\hline 2 & ART clinic & Positive & T. vaginalis & L2 confirmed & 12 & 98.3 \\
\hline 3 & ART clinic & Positive & Mycoplasma genitalium & L2 confirmed & 21 & 98.6 \\
\hline 4 & ART clinic & Positive & M.a genitalium & L2 confirmed & 72 & 99 \\
\hline 5 & ART clinic & Positive & T. vaginalis & Insufficient WGS read coverage & 0.5 & 29 \\
\hline 6 & STI clinic & Unknown & Neisseria gonorrhoeae & Insufficient clinical material & ND & ND \\
\hline 7 & STI clinic & Unknown & N. gonorrhoeae & Insufficient clinical material & ND & ND \\
\hline
\end{tabular}

was insufficient to allocate a biovar; insufficient DNA was available from 2 other samples for WGS.

This report shows emergence of $C$. trachomatis biovar L2 genital infection in women living in South Africa, a region to which lymphatic LGV is endemic (1). Instead of serologic analysis, we used molecular testing and WGS of clinical specimens to confirm the diagnosis, determine genetic relatedness, and identify the specific variant of genotype L. We observed LGV in specimens from women at the academic hospital in Pretoria, but not from women living in the Mopani District, $\approx 400 \mathrm{~km}$ away. Although the distribution of risk factors may be different, the close relatedness of LGV strains suggests that this might be a localized outbreak of genital $C$. trachomatis L2 infection among women living in Pretoria.

The clinical role of genital C. trachomatis biovar $\mathrm{L}$ infection in women remains to be determined. Analogous to non-LGV C. trachomatis infection in women and rectal LGV in MSM, the clinical spectrum of genital LGV in women may vary from a mucosal ulcer with intrapelvic lymphadenopathy to cervicitis with vaginal discharge, or it may manifest without any symptoms at all as persistent asymptomatic infection. Although rectal C. trachomatis infections have been reported in African women, the occurrence of rectal LGV is unknown (10).

The emergence of genital LGV in women poses a concern in our setting, which uses syndromic management for STIs, because it is unclear whether the infection would be treated adequately with the empirical regimen of azithromycin and ceftriaxone. The main limitation of this report is the lack of follow-up data to confirm whether syndromic management was effective for these biovar L C. trachomatis genital infections.

In conclusion, this report shows the emergence of genital C. trachomatis L2 infection in South African women. Further research about its distribution in the general population, clinical role, and the occurrence of rectal infections is warranted because it is unclear whether this STI is managed adequately under the current syndromic management guidelines.

\section{Acknowledgments}

We thank the students at the University of Pretoria and staff at the Anova Health Institute for their contributions to this project.
R.D. and the chlamydia genome sequencing were funded by the European Union FP7 PATHSEEK grant. We acknowledge the National Institute for Health Research University College London Hospitals/University College London Biomedical Research Centre and Medical Research Council-funded Pathogen Genomics Unit. J.B. receives additional funding from the National Institute for Health Research University College London Hospitals/University College London Biomedical Research Centre.

Dr. Peters is a clinical program specialist at the Anova Health Institute in South Africa. He is a professor of medical microbiology at the University of Pretoria and at Maastricht University. His research interest is the molecular epidemiology of infectious diseases, with specific focus on sexually transmitted infections.

\section{References}

1. Stoner BP, Cohen SE. Lymphogranuloma venereum 2015: clinical presentation, diagnosis, and treatment. Clin Infect Dis. 2015;61(Suppl 8):S865-73. http://dx.doi.org/10.1093/cid/civ756

2. de Vrieze NH, van Rooijen M, Speksnijder AG, de Vries HJ. Urethral lymphogranuloma venereum infections in men with anorectal lymphogranuloma venereum and their partners: the missing link in the current epidemic? Sex Transm Dis. 2013;40:6078. http://dx.doi.org/10.1097/01.OLQ.0000431359.26583.13

3. Heiligenberg M, Verweij SP, Speksnijder AG, Morré SA, de Vries HJ, Schim van der Loeff MF. No evidence for LGV transmission among heterosexuals in Amsterdam, the Netherlands. BMC Res Notes. 2014;7:355. http://dx.doi.org/10.1186/1756-0500-7-355

4. Gomes JP, Nunes A, Florindo C, Ferreira MA, Santo I, Azevedo J, et al. Lymphogranuloma venereum in Portugal: unusual events and new variants during 2007. Sex Transm Dis. 2009;36:88-91. http://dx.doi.org/10.1097/OLQ.0b013e31818b1e27

5. de Jesús De Haro-Cruz M, Deleón-Rodriguez I, Escobedo-Guerra MR, López-Hurtado M, Arteaga-Troncoso G, Ortiz-Ibarra FJ, et al. Genotyping of Chlamydia trachomatis from endocervical specimens of infertile Mexican women. Enferm Infecc Microbiol Clin. 2011;29:102-8. http://dx.doi.org/10.1016/ j.eimc.2010.08.014

6. Herida M, Kreplack G, Cardon B, Desenclos J-C, de Barbeyrac B. First case of urethritis due to Chlamydia trachomatis genovar L2b. Clin Infect Dis. 2006;43:268-9. http://dx.doi.org/10.1086/505310

7. Goldenberger D, Dutly F, Gebhardt M. Analysis of 721 Chlamydia trachomatis-positive urogenital specimens from men and women using lymphogranuloma venereum L2-specific real-time PCR assay. Euro Surveill. 2006;11:E061018.4.

8. Verweij SP, Catsburg A, Ouburg S, Lombardi A, Heijmans R, Dutly F, et al. Lymphogranuloma venereum variant L2b-specific polymerase chain reaction: insertion used to close an 
epidemiological gap. Clin Microbiol Infect. 2011;17:1727-30. http://dx.doi.org/10.1111/j.1469-0691.2011.03481.x

9. Christiansen MT, Brown AC, Kundu S, Tutill HJ, Williams R, Brown JR, et al. Whole-genome enrichment and sequencing of Chlamydia trachomatis directly from clinical samples. BMC Infect Dis. 2014;14:591. http://dx.doi.org/10.1186/s12879-014-0591-3

10. Peters RP, Dubbink JH, van der Eem L, Verweij SP, Bos ML, Ouburg S, et al. Cross-sectional study of genital, rectal, and pharyngeal Chlamydia and gonorrhea in women in rural South Africa. Sex Transm Dis. 2014;41:564-9. http://dx.doi.org/10.1097/ OLQ.0000000000000175

Address for correspondence: Remco P.H. Peters, University of Pretoria, Faculty of Health Sciences, Department of Medical Microbiology, Pathology Bldg, Rm 3-11, Private Bag X323, Pretoria, 0001, South Africa; email: rph.peters@gmail.com

\section{Unrecognized Dengue Virus Infections in Children, Western Kenya, 2014-2015}

\section{David M. Vu, Noah Mutai, Claire J. Heath, John M. Vulule, Francis M. Mutuku, Bryson A. Ndenga, A. Desiree LaBeaud}

Author affiliations: Stanford University School of Medicine, Stanford, California, USA (D.M. Vu, C.J. Heath, A.D. LaBeaud); Kenya

Medical Research Institute, Centre for Global Health Research, Kisumu, Kenya (N. Mutai, B.A. Ndenga); Technical University of Mombasa, Mombasa, Kenya (J.M. Vulule, F.M. Mutuku)

DOI: https://doi.org/10.3201/eid2311.170807

We detected a cluster of dengue virus infections in children in Kenya during July 2014-June 2015. Most cases were serotype 1 , but we detected all 4 serotypes, including coinfections with 2 serotypes. Our findings implicate dengue as a cause of febrile illness in this population and highlight a need for robust arbovirus surveillance.

$\mathrm{D}$ ue to lack of national surveillance programs, the extent of infection with dengue viruses (DENV) among children is largely unknown in much of sub-Saharan Africa (1). Uncovering this hidden burden is critical for making informed public health decisions that affect populations that are most vulnerable to vectorborne disease. To address this knowledge gap, in January 2014, we initiated a 4.5-year study of the transmission and extent of arbovirus infection in children in Kenya. Although the study continues through June 2018, we identified a cluster of DENV infections among febrile children in western Kenya, prompting this report to raise awareness of DENV as a cause of acute febrile illness (AFI) among children in this country.

We describe results from a cohort of children with AFI who came to 1 of 2 regional health centers that serve the communities of Chulaimbo (a rural village) and Kisumu (an urban city), both located in western Kenya. The cohort is part of a larger ongoing parent study that enrolls additional child cohorts at other study sites or with other study designs and collects vector and environmental data. Details of the parent study are beyond the scope of this report and will be described elsewhere. The study is being conducted under the supervision of the institutional review board of Stanford University (Stanford, CA, USA; IRB-31488) and the scientific and ethics review unit of the Kenya Medical Research Institute (Kisumu, Kenya; SSC 2611).

For this study, we enrolled febrile children 1-17 years of age. At the enrollment and 1-month follow-up visits, we collected information on demographics and risk factors, performed a physical examination, and obtained blood samples. We tested the blood samples for DENV RNA by reverse transcription PCR (RT-PCR) (2).

We enrolled 1,258 children with AFI during January 2014-April 2016. We tested blood samples from 1,104 study participants $(87.8 \%)$ by RT-PCR. Of those, $82(7.4 \%)$ were positive for DENV RNA: 58 (70\%) were serotype 1 (DENV1), 2 (2.4\%) were serotype 2 (DENV-2), 13 (15.9\%) were serotype 3 (DENV-3), and 2 (2.4\%) were serotype 4 (DENV-4) (Figure). We also detected co-infection with 2 serotypes in 6 participants: 2 children had DENV-1 and $-3 ; 3$ children had DENV-1 and -4; and 1 child had DENV-2 and -3 .

Most DENV cases occurred during July 2014-June 2015. The likelihood of testing positive for DENV by PCR did not differ by age or sex. The median age of DENV PCRpositive participants was 3.5 years (IQR 2.0-5.2 years); $43.9 \%$ of participants were female. We did observe a higher likelihood of testing positive by PCR for children from the rural site, Chulaimbo (9.6\%), compared with those from the urban site, Kisumu (5.8\%) ( $p=0.017$ by Fisher exact test; odds ratio $1.97,95 \%$ CI $1.14-3.43$, adjusted for age and sex). Continued surveillance is needed to investigate whether DENV is endemic to these areas and if there are differences in regional endemicity.

Although reports of DENV infection in Kenya remain sparse, several sero-epidemiologic studies have produced evidence of DENV transmission by demonstrating serum DENV IgG in patients of all ages (3-6). In 2013, an outbreak of DENV-2 in Mombasa was detected, in part, by RTPCR (7). More recently, DENV infections were described in 43 adult patients with fever who resided in Mtwapa, on the coast of Kenya near Mombasa; these cases occurred February 2014-January $2015(8)$, which overlapped with the period in which we observed the DENV cases we report in this 